\title{
ERRATUM
}

\section{Early pathogenic events associated with Sjögren's syndrome (SjS)-like disease of the nod mouse using microarray analysis}

Smruti J Killedar, Sarah E Eckenrode, Richard A McIndoe, Jin-Xiong She, Cuong Q Nguyen, Ammon B Peck and Seunghee Cha

Laboratory Investigation (2007) 87, 398. doi:10.1038/labinvest.3700542

Correction to: Laboratory Investigation (2006) 86, 1243-1260. doi:10.1038/labinvest.3700487

Following the publication of the above paper, an error was identified in the name of one of the authors. The correct authors' names are shown above. 